\title{
A Complex Approach to Glossalgia Treatment Based on the Current Data on the Specificity of lis Etiopathogenesis
}

\author{
DOI: $10.17691 / \mathrm{stm} 2018.10 .2 .15$
}

Received March 27, 2017

L.K. Ellaryan, MD, PhD, Associate Professor, Department of Propaedeutic Dentistry;

L.N. Kazarina, MD, DSc, Professor, Head of the Department of Propaedeutic Dentistry;

E.A. Shevchenko, MD, DSc, Professor, Department of Pathological Physiology

Privolzhsky Research Medical University, 10/1 Minin and Pozharsky Square, Nizhny Novgorod, 603005, Russia

The aim of the study was to assess the efficacy of a novel complex approach to glossalgia treatment based on the current data on the specificity of its etiopathogenesis.

Materials and Methods. 58 patients with glossalgia aged 42-55 years have been examined and treated. The condition of the capillary blood flow was determined by laser Doppler flowmetry. At the laboratory stage, such indices of the local immunity in the oral cavity as slgA, IgA, IgG, and lysozyme have been investigated, and the coefficient of local immunity factors balance has also been calculated.

Results. Of 58 patients receiving our complex etiopathogenetically justified treatment, complete recovery has been reached in 39 individuals and significant improvement in 19.

Conclusion. Introduction of preparations improving microcirculation and normalizing metabolic processes in glossalgia treatment results in more rapid and long-term effect.

Key words: glossalgia; glossalgia treatment.

\section{Introduction}

Glossalgia, a disease manifesting itself in painful and burning sensations of the tongue, is considered the most ambiguous in understanding and difficult in therapy neurostomatological pathology. It is characterized by pain in the mouth without any signs of inflammation and specific injuries. Clinical heterogeneity realized at the level of comorbidity with a somatic pathology is typical of glossalgia [1-3].

Advances of recent years in such related to medicine sciences as biochemistry, neurophysiology, neurology, and psychiatry provide the possibility to widen the notion of the pathogenesis of neurostomatological diseases [4-6].

A great number of works have been devoted to etiology, pathogenesis, and methods of glossalgia treatment but there is no agreement on this problem. Some authors believe this entity to be of a polyetiologic character proving their point of view by the presence of such factors in the clinical picture as dishormonemia, alterations of the nervous system functions, diseases of gastrointestinal tract and cardiovascular system, and so on [7-9]. On this basis, they believe that treatment of glossalgia should be symptomatic, i.e. oriented to the correction of the changes that are revealed in this illness.
Even when local procedures are used (blockades, applications, physiotherapy) symptomatic treatment remains ineffective [10].

Other authors relying on the latest notion about the pathogenesis of tongue diseases think that an important role in glossalgia onset is played by general factors such as disorders in the system of immuno- and neuroregulation. These mechanisms have been poorly studied so far and the assessment of their role is rather vital [11].

The results of glossalgia treatment are at present disappointing. Even up-to-date diagnostic methods and adequate therapy ensure the success of treatment only in $32 \%$ of cases.

The analysis of the current literature data allows us to assume that all groups of factors leading to glossalgia are only provocating. An actual causative factor is the condition of the tongue itself together with the structures providing it with humoral and neurotrophic substrates. It is indirectly testified by the fact that glossalgia does not appear in many people with significantly marked provocative factors which can be in combination with each other.

We suppose that multiple investigations of neurological status, biochemical, immunological, and other indices in glossalgia are rather aimed at

Corresponding author: Elena A. Shevchenko, e-mail: el.shevchenko2010@yandex.ru 
studying important links of pathogenesis than at the very cause of the disease which still remains obscure. Therefore, methods of prevention and treatment used must be oriented to separate pathogenic links of this disease. And as many systems of the body participate in the pathogenesis, consequently, the approach to the treatment should be complex.

Taking into consideration current data on the mechanisms of the pathogenesis there has been developed a novel approach to glossalgia management which consists in using immune- and neuroregulating agents for its correction.

The aim of the study was to assess the efficacy of a novel complex approach to glossalgia treatment based on the current data on the specificity of its etiopathogenesis.

\section{Materials and Methods}

The present investigation was conducted on the basis of the Departments of Propaedeutic Dentistry and Pathological Physiology of Privolzhsky Research Medical University of Nizhny Novgorod. 58 patients with glossalgia ( 38 women and 20 men) have been examined and treated, 30 practically healthy persons served as a control group (15 women and 15 men aged $42.3 \pm 5.8$ years). 57 examined patients suffered from the diseases of gastrointestinal tract and liver, 50 patients had cardiovascular pathology, 19 - with endocrine disorders.

The diagnosis of glossalgia was established in accordance with the diagnostic criteria of the ICD-10, International Association for the Study of Pain (1994), International Headache Society (1996).

While consulting the patients the dentist obtained their complaints and medical history, carried out clinical, instrumental, and objective examination.

The examined patients presented the following complaints: heaviness and "tiredness" of the tongue especially under the intensive speech load, numbness, "contraction" of the tongue, "tingling", "burning", pain, paresthesia, and dry mouth. Paresthetic and pain sensations did not have distinct borders and were of temporal character. According to the history data, they disappeared during food intake, unpleasant sensations were often absent in the morning or were slightly felt, became more intensive by the evening or during a longterm conversation, nervous excitation. Various dental manipulations were noted in some medical histories which in some cases resulted in pains and paresthesias in the tongue.

The present retrospective study was approved by the Ethics Committee of Privolzhsky Research Medical University and complied with the Declaration of Helsinki (2013). Written informed consent was obtained from every patient for the scientific analysis of their data.

Laser Doppler flowmetry was used to assess the condition of capillary blood flow in the tongue in patients with glossalgia and in the control group, as in glossalgia at the moment of pain attack there may be a shortterm disorder of cerebral circulation, ischemia, which may result in small arteries constriction, decrease of functioning capillaries, reduction of the volumetric and linear blood flow rate.

The laboratory stage of the study included identification of indices of local oral immunity: levels of $\lg A, \lg G$, secretory $\lg A$ (slgA), lysozyme as well as the coefficient of local immunity factors balance developed by V.G. Dorofeichuk and N.I. Tolkachyova (1987). For this purpose, $3-5 \mathrm{ml}$ of fasting oral fluid was collected in the morning without stimulation. The sample was stored in a fridge at $-20^{\circ} \mathrm{C}$. The slgA level in the oral cavity was identified by a gel radial immunodiffusion assay according to G. Mancini, A. Carbonara (1965) using the methodological recommendations offered by E.V. Chernokhvostova and S.I. Golderman (1975). Oral lysozyme was determined using photonephelometric method. Coefficient of local immunity factors balance was used for integral evaluation of the local immunity condition in the oral cavity. Serum immunoglobulins $(\lg A$, $\lg \mathrm{G}$ ) in the oral fluid were also quantitatively determined by the same gel radial immunodiffusion assay.

Statistical processing of the results was performed by the methods of assessing the reliability of the results and variation statistics. The data obtained were processed and analyzed by means of Excel, Stadia, Statistica 7.0 programs.

\section{Results and Discussion}

The analysis of the immunological investigation results showed that in all patients with glossalgia lysozyme concentration in saliva was decreased and IgG concentration was statistically significantly increased. $\lg$ A concentration was also significantly reduced in all patients while slgA only in the persons with concomitant diseases. Both these immunoglobulins reflect not only the state of the local but the general immunity as well. Since dysgammaglobulinaemia was revealed in all patients, its availability should be considered a characteristic feature of glossalgia.

After the treatment, the level of lysozyme, IgA, and $\operatorname{sig} A$ grows and $\lg G$ reduces. Many cytokines are mediators of both immune and neuroendocrine systems therefore they are likely to participate in the pathogenesis of glossalgia. Further investigations are necessary in this direction.

Taking into account the latest knowledge on pathogenetic links of glossalgia and the data obtained, the authors have elaborated a novel complex approach to the treatment of this disease. It includes a pathogenetic treatment along with etiotropic, symptomatic, and sanogenetic therapy, and implies differential approach to the treatment of various groups of patients.

Etiopathogenetically oriented treatment of glossalgia consists in elimination of provoking factors and triggering mechanisms which is achieved by the agents acting on 
the tissue metabolism and normalizing patient's psychoemotional sphere.

To correct such pathogenetic factors as microcirculation disorder, hypoxia, angiospasms, and thrombocyte hyperaggregation, it was decided to complement the treatment by the preparations for metabolic process normalization, by vascular medications improving cerebral circulation, and medications for neuroregulation.

To provide therapy improving cerebral circulation and metabolism, regulating general metabolism, reducing psycho-emotional stress and vegetovascular disorders, normalizing and activating the processes of defensive inhibition in CNS and microcirculation in all tissues, patients were randomly divided into three groups with the following administrations:

group 1 - Cortexin, intramuscular injections, $2 \mathrm{ml}$ per day for 10 days and Combilipen, intramuscular injections, $2 \mathrm{ml}$ per day for 10 days;

group 2 - Vinpocetine, 1 tablet $(0.05 \mathrm{~g}) 3$ times a day for 1 month in combination with Alka-Prim, 1 tablet $(0.1 \mathrm{~g})$ sublingually 3 times a day for 1 month; Neuromultivit, 1 tablet twice a day for 1 month;

group 3 - Cerebrolysin, intramuscular injections, $2 \mathrm{ml}$ during 10 days; Milgamma, intramuscular injections, $2 \mathrm{ml}$, once a day during 10 days.

Medications containing vitamins of group B were administered to the patients of all three groups. Active participation of vitamin $B_{1}$ in the processes of excitement conduction in synapses as well as the necessity of vitamin $B_{6}$ serving as a coenzyme of the most essential nerve tissue enzymes and participating in biosynthesis of neuromediators (adrenaline, noradrenaline, dopamine) were taken into consideration.

The set of medications used in group 1 demonstrated the best results being in agreement with the data of some Russian scientists. A slightly weaker positive effect was achieved with scheme 3 (group 3) preferably used abroad. The lower effect is likely to be connected with the fact that Cerebrolysin contains only $15 \%$ of neuropeptide fractions compared to Cortexin. Modern neurochemistry proved that neuropeptides bear the main neurotrophic pharmacological load.

At present, there appeared a new drug, Cerebrolysin EO21, enriched with peptides up to $25 \%$ which shows in experiments a greater clinical effect than Cerebrolysin. Further investigations in this direction will show the actual efficacy of this medication.

Scheme 2 (group 2), in our opinion, is indicated to the patients with the least prominent manifestations of clinical semiotics. Its effect is not so evident compared to schemes 1 and 3 (groups 1 and 3).

To eliminate inner psychological conflict and mobilize natural resources of the patients to fight glossalgia, our doctors discussed the disease with the patients and their relatives in order to change psychologically the habitual "pain" behavior.
Individual therapeutic physical training was recommended to increase physical activity for improving microcirculation, normalizing patients' psychological background and social adaptation which, in turn, activates the processes of sanogenesis in this pathology.

Patients were examined twice: before and after the treatment to assess the efficacy of the conducted glossalgia therapy. The results have been confirmed statistically $(p<0.05)$. The obtained clinical indices of patients' condition were analyzed and compared with the control values.

After the treatment, the intensity of pains and parsthesias in all patients participating in the study underwent significant changes. If a mean value of pain according to the visual analogue scale in glossalgia was $5.50 \pm 0.03$ points prior to the treatment, after the course of therapy this value reduced essentially to $1.70 \pm 0.03$ points $(p<0.05)$. Statistically significant reduction of pain intensity measured by the visual analogue scale confirms the efficacy of the administered treatment.

After the complex glossalgia therapy, positive dynamics of microcirculation parameters according to the data of laser Doppler flowmetry was characterized by normalization of all indices (see the Table).

Statistically significant $(p<0.01)$ decrease of microcirculation index and increase of the mean square deviation (of the flux) and the coefficient of variation due to the reduction of congestive phenomena in the venular microcirculation link and intensification of tissue perfusion with blood after the treatment were noted. Improvement of microcirculation and vasomotor activity of microvessels was also noted. Enhancement of the flux is caused by a more intensive functioning of the mechanisms regulating microcirculation. Besides, increase of the coefficient of variation, being an integral assessment of perfusion, also reflects microcirculation improvement. These changes were felt in the reduction of pains and paresthesias in the tongue.

Treating patients with glossalgia one should take into account the fact that a high percentage of periodontium diseases (20-66\%), low-quality fillings, and orthopedic constructions (8-30\%) are noted in their dental status. In this connection, all patients underwent professional hygienic procedures and sanation of the oral cavity, proper prosthetic treatment was also recommended.

Our complex pathogenetically justified approach to treating patients with glossalgia allowed us to achieve
Integrated values of laser Doppler flowmetry indices in patients with glossalgia before and after the treatment $(M \pm m)$

\begin{tabular}{lccc}
\hline \multicolumn{1}{c}{ Indexes } & \multicolumn{3}{c}{ Glossalgia } \\
\cline { 2 - 4 } & Before therapy & After therapy & Control \\
\hline Microcirculation (perf. units) & $13.62 \pm 0.20^{*}$ & $10.34 \pm 0.12^{*}$ & $9.12 \pm 0.02$ \\
\hline Mean square deviation (flux) & $0.60 \pm 0.03^{*}$ & $0.71 \pm 0.01^{*}$ & $0.93 \pm 0.06$ \\
\hline Coefficient of deviation & $7.31 \pm 0.03^{*}$ & $9.49 \pm 0.02^{*}$ & $10.14 \pm 0.02$ \\
\hline
\end{tabular}

${ }^{*} p<0.01$ compared to the control. 
the following results: of 58 patients complete recovery has been reached in 39 individuals $(67.2 \%)$ and significant improvement in 19 (32.8\%). Stable remission was observed during two years of dynamic follow-up.

Thus, application of etiopathogenetically justified pharmacological scheme including Cortexin and Milgamma showed not only the most prominent improvements of clinical and laboratory indices but immune as well: reduction of coefficient of local immunity factors balance was observed already by day 6 , and on days 14 and 30 progressive decrease of coefficient of local immunity factors balance continued.

\section{Conclusion}

Specifics of etiology and pathogenesis of glossalgia should be taken into consideration in the complex approach to the treatment of this disease. This approach must be directed to normalization of psycho-emotional state, functions of internal organs with obligatory introduction of medications improving trophism of the tongue and creating conditions for normalizing local immunity and the state of the microcirculatory bloodstream. It is preferable to use a complex of drugs including Milgamma, Cerebrolysin, and Combilipen. In less severe cases, Vinpocetine, Alka-Prim, and Neuromultivit should be administered.

Study Funding and Conflicts of Interest. The study was not supported by any sources. The authors have no conflicts of interest to disclose.

\section{References}

1. Kunin A.A., Borisova E.G., Pankova S.N., Kumirova O.A., Lepekhina L.I., Shelkovnikova S.G., Erina S.V., Belenova I.A. Klinicheskie aspekty differentsial'noy diagnostiki i lecheniya osnovnykh zabolevaniy yazyka [Clinical aspects of differential diagnosis and treatment of major diseases of the tongue]. Voronezh; 2013.

2. Borisova E.G. Clinical and anamnestic analysis of patients with chronic pain syndromes of the tongue. Rossiyskiy zhurnal boli 2014; 1: 29-30.

3. Borisova E.G. The modern view on quality of diagnosis of chronic pain syndromes in tongue. Fundamental'nye issledovaniya 2014; 7(2): 246-249.

4. Jääskeläinen S.K. Pathophysiology of primary burning syndrome. Clin Neurophysiol 2012; 123(1): 71-77, https://doi. org/10.1016/j.clinph.2011.07.054.

5. Uspenskaya O.A., Shevchenko E.A. A study of the influence of a range of medical products on the clinical course of local inflammatory processes in the buccal cavity and urogenital tract at pregnant women with CPI. Fundamental'nye issledovaniya 2015; 1-4: 837-839.

6. Zhulev E.N., Uspenskaya O.A., Shevchenko E.A., Alexandrov A.A. Assessment of orthopedic treatment effect on cerebral hemodynamics in patients with discirculatory encephalopathy. Sovremennye tehnologii v medicine 2013; 5(1): 82-85.

7. Shevchenko E.A., Uspenskaya O.A., Kondyurov I.M., Kurylev V.V., Rossohin V.F. The estimation of a virus component for diagnosis and treatment of oral inflammatory diseases. Sovremennye tehnologii v medicine 2012; 3: 96-99.

8. Kazarina L.N., Bolsunovsky S.M. Effect of statins on the microcirculation of periodontal tissue in patients with metabolic syndrome. Meditsina 2016; 2: 43-50.

9. Komiyama O., Nishimura H., Makiyama Y., lida T., Obara R., Shinoda M., Kobayashi M., Noma N., Abe O., De Laat A., Kawara M. Group cognitive-behavioral intervention for patients with burning mouth syndrome. J Oral Sci 2013; 55(1): 17-22, https://doi.org/10.2334/josnusd.55.17.

10. Rodriguez-Cerdeira C., Sanchez-Blanco E. Treatment of burning mouth syndrome with amisulpride. J Clin Med Res 2012; 4(3): 167-171, https://doi.org/10.4021/jocmr972w.

11. Tiunova N.V. Conceptual bases of non-invasive neuromodulation in treatment of burning mouth syndrome. Dental Forum 2016; 4(63): 69-70. 